\title{
RESONANCIA MAGNETICA MAMARIA: INDICACIONES POCO HABITUALES*
}

Drs. Paulina González $M^{(1,2)}$, Eleonora Horvath $P^{(2)}$, Patricia Arancibia $H^{(1)}$, María Galleguillos $P^{(2)}$, Marcela Jacard $C^{(1)}$, Alejandra López $P^{(1)}$, Paulina Neira $V^{(1)}$, Dulia Ortega $T^{(1)}$, Miguel Pinochet $T^{(2)}$, Teresa Taub $E^{(1)}$, Marcela Uchida $S^{(2)}$, Heriberto Wenzel $K^{(2)}$.

1. Centro de Imagenología del Hospital Clínico de la Universidad de Chile.

2. Servicio de Imagenología Mamaria de la Clínica Alemana de Santiago.

* Trabajo premiado por el Comité de Selección en el Congreso de Radiología 2005

\begin{abstract}
The indications of breast MRI are now clearly established and internationally accepted. They are also applied in Chile as was agreed in the last the National Consensus on the management of breast cancer. In our study the indications not mentioned in the consensus were classified as infrequent. We determined that a $9.2 \%$ of breast MRI performed in our study centers were due to infrequent indications, leading to a change in medical management in $71,4 \%$ of these patients, although this change sometimes had no scientific evidence availing it.

These results suggest that MRI could be useful in specific situation and reveal a need for multicentric high quality studies in order to gather enough evidence to justify its use in indications other than the ones already established. In other situations MRI would not be recommended. In exceptional cases, where it seems useful to perform this type of exam it is suggested to make the advisable to make the decision after discussion in a interdisciplinary committee.
\end{abstract}

Key words: Breast, Magnetic resonante imaging.

Resumen: Las indicaciones de resonancia magnética mamaria están bien definidas $e$ internacionalmente aceptadas. Se aplican también en Chile según lo establecido en el último Consenso Nacional sobre cáncer mamario, sin embargo observamos que un porcentaje no despreciable (9.2\%) de las resonancias mamarias realizadas en nuestros centros de atención son por indicaciones distintas a las establecidas. En un $71.4 \%$ de estos casos generó un cambio en la conducta médica, situación que en algunos casos no contó con respaldo científico establecido. Estos resultados orientan a pensar que la resonancia sería de utilidad

González P, y cols. Resonancia magnética mamaria: Indicaciones poco habituales. Rev Chil Radiol 2005; 11: 161-165.

Correspondencia: Dra. Paulina González M. E-mail:pgonzalezm@alemana.cl en casos especificos y demuestran la necesidad de estudios sistematizados, multicéntricos que lleven a reunir la evidencia suficiente para justificar su uso en indicaciones distintas a las ya aceptadas.

En los casos en que parezca conveniente realizar este tipo de estudio, sería recomendable discutir su eventual utilidad e influencia en la conducta clínica, en el seno de equipos de especialistas multidisciplinarios.

Palabras clave: Mama, Resonancia magnética.

\section{Introducción}

En el estudio de patología mamaria, la resonancia magnética $(\mathrm{RM})$ es una técnica de diagnóstico por imágenes, que complementa a la mamografía y a la ecografía y que está en uso desde hace menos de una década en Chile. Es un examen extremadamente sensible, pero poco específico en la detección del cáncer.

En la II Jornada Chilena de Consenso de Cáncer en Mama, realizada en Viña del Mar, en el año 2003, se estableció su utilidad en la detección y control del cáncer mamario, determinándose las siguientes indicaciones:

1. Evaluación preoperatoria para determinar extensión tumoral.

2. Detección precoz de recidiva en mama tratada.

3. Detección de neoplasias ocultas en presencia de metástasis axilares.

4. Monitoreo de quimioterapia neoadyuvante.

Fuera de la esfera oncológica se encuentra ampliamente reconocida como el examen de elección en la evaluación de las prótesis mamarias y sus complicaciones.

Cabe destacar que en nuestro país no existen estudios sistematizados que validen su uso en indicaciones distintas a las ya establecidas y que en este estudio hemos denominado como poco habituales.

\section{Objetivos}

Determinar las indicaciones de RM mamaria no consensuadas y que se efectúan en la práctica 
cotidiana, evaluar el porcentaje de realización en este estudio bi-céntrico y su influencia en el manejo clínico de los pacientes.

\section{Materiales y método}

Se realizó un estudio descriptivo, retrospectivo, que consistió en la revisión de la totalidad de las RM mamarias realizadas en el Centro de Imagenología del Hospital Clínico de la Universidad de Chile (Centro 1) y en el Servicio de Imagenología Mamaria de la Clínica Alemana de Santiago S.A. (Centro 2) desde enero de 2004 a julio de 2005. Se estableció el motivo de realización de todas ellas y posteriormente se efectuó seguimiento a aquellas pacientes con indicaciones consideradas como poco habituales a fin de evaluar si este examen implicó un cambio en la conducta médica.

Se estableció en cada caso, cuál habría sido el manejo según la categoría BI-RADS de las imágenes previas a la realización de la RM y consideramos un cambio de conducta cuando esto no se cumplió.

En ambos centros el examen se realiza con el paciente en decúbito ventral, con bobinas de superficie dedicada en secuencias T2 y T1 dinámicas pre y post administración de medio de contraste paramagnético (Gadolinio, $0,1 \mathrm{ml} / \mathrm{kg}$ ), con sustracciones. En el post-procesamiento se realizaron reconstrucciones MIP y MPR.

En el Centro 1 se utiliza un protocolo de estudio axial con técnica PAT en secuencias T2 TSE sin saturación grasa, con cortes de $5 \mathrm{~mm}$ y secuencias T1 dinámicas VIBE. En el post procesamiento se realiza evaluación de curvas cinéticas. Se utilizó una matriz de 512 x 512 .

En el Centro 2 el estudio se realiza en planos coronales, en secuencias T2 con saturación grasa, con cortes de $3 \mathrm{~mm}$ ZIP 2 y secuencias T1 dinámicas 3D FAME. La bobina es Phased Array de 4 canales, la matriz de adquisición es $256 \times 256$ ZIP 512. Se otorga mayor importancia a la evaluación de la morfología de las lesiones que a las curvas cinéticas.

En presencia de silicona se agregó secuencias STIR axiales y sagitales con pulsos de saturación de agua en cortes de $4 \mathrm{~mm}$ de grosor.

Todos los exámenes fueron informados por médicos radiólogos experimentados en imágenes mamarias.

\section{Resultados}

Durante un período de 19 meses, en ambos centros se realizaron $356 \mathrm{RM}$ mamarias. De éstas $33(9.2 \%)$ fueros realizadas por indicaciones poco habituales. Se logró seguimiento en 24 de estos pacientes que constituyen el universo de este estudio.

En ocho casos la indicación fue una distorsión de la arquitectura mamográfica, constituyéndose en la indicación más frecuente. En cinco de estas pacientes la RM fue negativa; en ellas se mantuvo manejo expectante, un caso corresponde a una cicatriz radiada según la biopsia Core realizada bajo US. En los tres casos restantes la RM demostró captación focal, en uno de ellos se realizó una biopsia estereotáxica con resultado histológico benigno y en las dos restantes biopsias radio-quirúrgicas, ambas con resultado benigno, una de ellas por cicatriz radiada.

En seis casos la RM fue realizada por presencia de nódulos. En dos pacientes por nódulos múltiples y en cuatro por nódulo solitario, dos de categoría BI-RADS 3 y dos de categoría BI-RADS 4 según su aspecto ecográfico. Ninguno de los nódulos únicos tuvo representación en la resonancia, no obstante, los de categoría BI-RADS 3 fueron sometidos a biopsia; cuyo resultado fue benigno. Los nódulos catalogados como BI-RADS 4, están bajo seguimiento ecográfico.

De las pacientes con nódulos múltiples en una se indicó la RM por el aspecto hipervascularizado de las lesiones al Doppler color, dado que éstos no presentaron refuerzo con el gadolinio en RM, se indicó control. La otra paciente tenía antecedentes de un tumor Phyllodes operado en la mama izquierda y múltiples nódulos bien delimitados en la mama derecha. La RM demostró que uno de estos nódulos presentaba impregnación con el medio de contraste con curva cinética tipo lavado, por lo que se realizo biopsia y posterior cirugía que demostró un tumor Phyllodes (Figura 1).

En un caso de asimetría focal, sin representación ecográfica, la RM no demostró signos de malignidad, se realizó control con mamografía y ecografía cada 6 meses.

En un caso la RM fue solicitada por micro calcificaciones BI-RADS 4 cuya biopsia percutánea previa resultó una lesión mucocele like. El examen fue normal y quedó en control cada seis meses.

En un caso el examen fue indicado por retracción cutánea, en mamas densas normales $\mathrm{BI}$ RADS 1 en mamografía y ecografía normal, sin lesión palpable. En este caso se demostró dos nódulos BIRADS 3 en localización distinta a la zona de retracción. La paciente se manejó en forma expectante, con control.

En un caso el estudio fue realizado por ansiedad de la paciente por antecedentes familiares de cáncer de mama, sin signos ecográficos ni mamográficos sospechosos de malignidad. La resonancia identificó un nódulo de aspecto benigno, no obstante, fue biopsiado. La histología resultó benigna.

En dos pacientes la RM fue realizada por mamas densas ACR 4 y un área hipoecogénica a la ecografía. En ambos casos el examen fue normal y se optó por realizar control anual. Una de ellas tenía ya una biopsia core que demostraba adenosis. 
En un caso se solicitó el estudio por un quiste complejo a la ecografía. La resonancia no demostró impregnación patológica. No se realizó biopsia.

Además hubo tres pacientes con antecedentes de inyección libre de biopolímeros en las mamas en quienes no se evaluó cambio de conducta puesto que no existe consenso sobre el manejo clínico en esta situación específica. En una de ellas, que consultó por eritema cutáneo, la RM demostró infiltración mamaria por cuerpo extraño y celulitis. En otra paciente que consultó por nódulos palpables, la RM demostró múltiples nodulillos subcutáneos con aspecto de biopolímeros. La tercera paciente fue derivada por una masa palpable, en ella se demostró una masa hipercaptante, altamente sospechosa, que resultó ser un carcinoma ductal infiltrante, no visible en la mamografía ni ecografía. La paciente fue sometida a mastectomía (Figura 2).

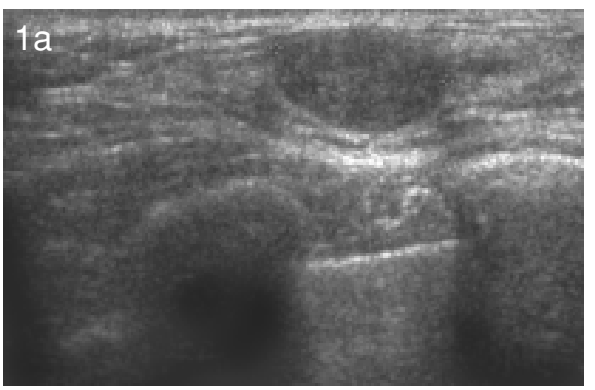

Figura 1 a, b. Paciente con antecedentes de un tumor phyllodes operado en la mama izquierda. a) La ecografía demostró múltiples nódulos bien delimitados en la mama derecha. b) La RM demostró que uno de estos nódulos presentaba captación del medio de

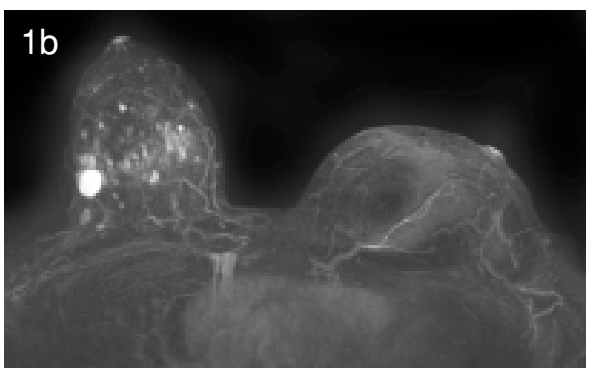
contraste con una curva de lavado, por lo que se realizo biopsia y posterior cirugía que demostró un tumor phyllodes.

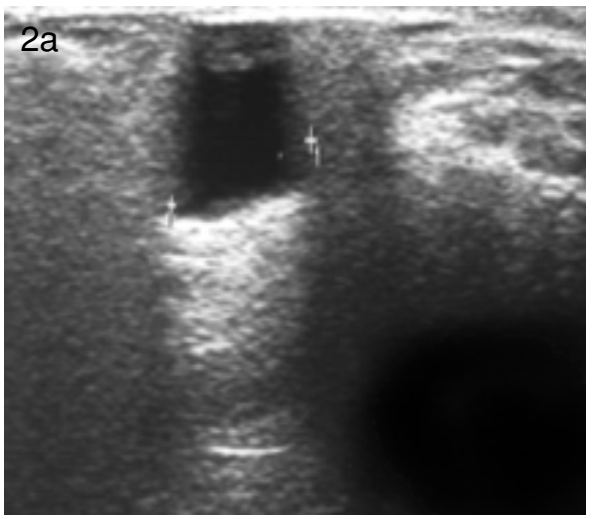

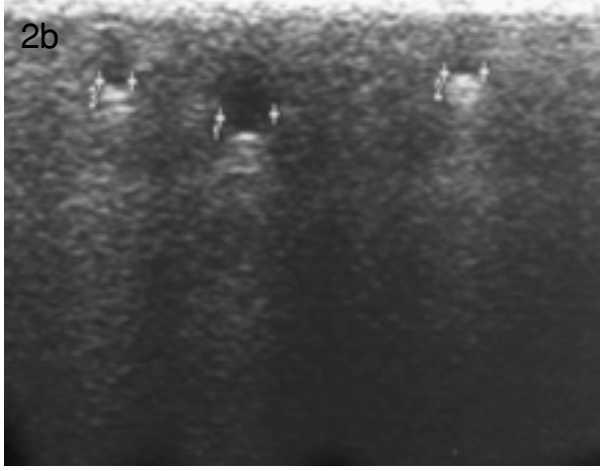
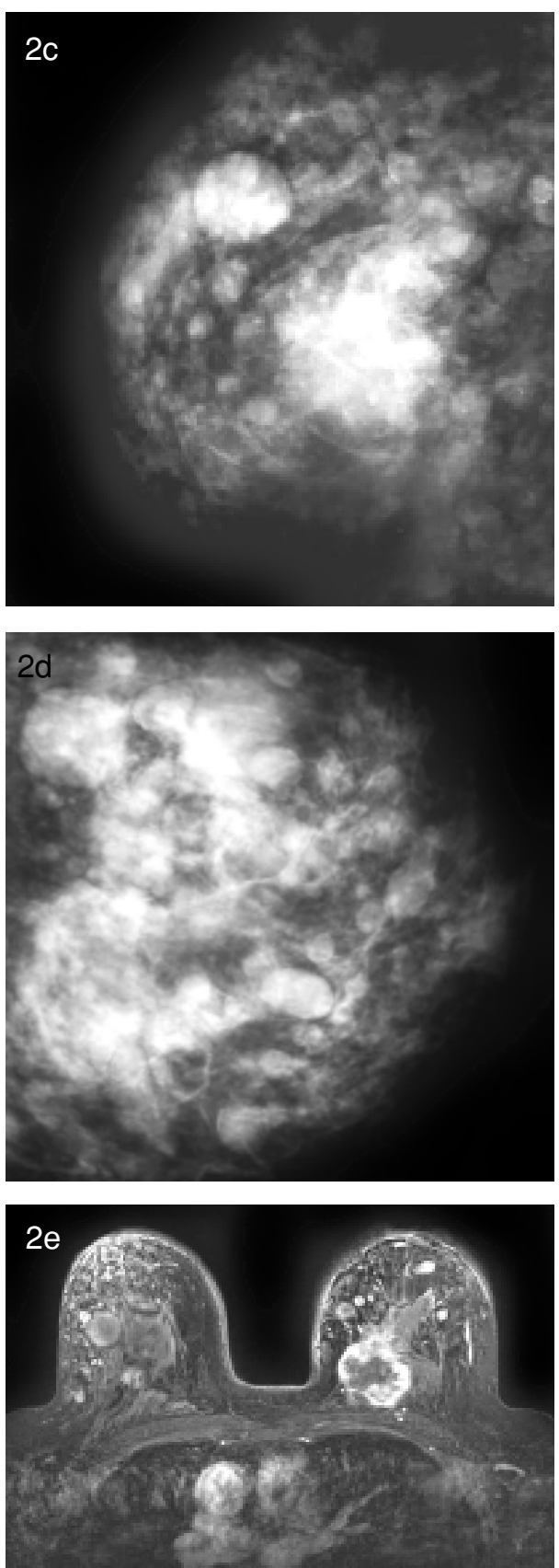

Figura 2 a-e. Paciente con antecedentes de inyección libre de biopolímeros, enviada a estudio por una masa palpable en la mama izquierda. a,b) La ecografía demostró una importante atenuación del sonido e imágenes quísticas compatibles con siliconomas. c, d) La mamografía evidenció la presencia de múltiples nódulos bilaterales densos, la mayoría bien definidos. e) La RM mamaria evidenció que la masa palpable correspondía a una lesión hipercaptante, altamente sospechosa, que resultó ser un carcinoma ductal infiltrante. La paciente fue sometida a mastectomía. 
De las 21 pacientes en quienes se evaluó cambio en la conducta médica, la RM implicó un cambió en la conducta en 15 casos, es decir, en un $71.4 \%$ (Tabla I).

\begin{tabular}{|c|c|c|}
\hline Indicación & $\mathbf{N}^{\circ}$ & Cambio manejo \\
\hline Distorsión arquitectura & 8 & 7 \\
\hline Nódulos & 6 & 4 \\
\hline Área hipoecogénica & 2 & 1 \\
\hline Asimetría focal & 1 & 1 \\
\hline Quiste complejo & 1 & 1 \\
\hline Mucocele like & 1 & 0 \\
\hline Retracción cutánea & 1 & 0 \\
\hline Ansiedad & 1 & 0 \\
\hline Inyección biopolímeros & 3 & No evaluable \\
\hline TOTAL & 24 & 15 \\
\hline
\end{tabular}

\section{Discusión}

Las indicaciones de RM mamaria hoy están bien definidas e internacionalmente aceptadas. Se aplican también en Chile según lo establecido en el último Consenso Nacional sobre el manejo del cáncer mamario.

Analizados nuestros resultados, nos demuestran que en los casos de nódulos únicos y/o áreas hipoecogénicas visibles en US, la RM no aportó información adicional, sólo costo suplementario. Estas lesiones se pueden manejar con biopsia percutánea guiada por US, o con seguimiento, según sea el caso.

En nódulos múltiples su utilidad es discutible. Sería beneficiosa en el caso de pacientes de alto riesgo, en quienes se hace necesario decidir la realización de estudio histológico. Un patrón de captación de carácter sospechoso de uno de ellos permitiría dirigir la biopsia. Pero, por otra parte, un análisis acucioso de las características ecográficas y el control de evolución también permitirían seleccionar aquellas lesiones que necesiten análisis histológico.

No creemos conveniente realizar RM mamaria en caso de un quiste complejo. Una punción con aguja fina hubiera sido suficiente para distinguirlo de un quiste complicado y valorar así la necesidad de escisión quirúrgica.

La paciente con diagnostico de mucocele like en la biopsia percutánea, incluso con RM sin captación patológica, debería haber ido a cirugía ya que este tipo de lesión se asocia a la existencia de cáncer.
En las distorsiones de la arquitectura mamográficas, sin representación en ecografía, fue donde la resonancia tuvo la mayor implicancia en cambiar la conducta médica, ya sea determinando espaciamiento de los controles o variando el tipo de biopsia a utilizar. Otra utilidad descrita en la literatura es para las distorsiones de arquitectura, visibles en una sola proyección mamográfica, sin representación ecográfica, lesiones que sólo sería posible marcar o biopsiar con estereotaxia. En estos pacientes la RM permite a veces encontrar y biopsiar la lesión, sin embargo, hasta la fecha de esta publicación, no existen sistemas compatibles para marcación o biopsia bajo resonancia en nuestro país. En nuestra experiencia, una ecografía dirigida, posterior a la RM y guiada por sus hallazgos, permite solucionar la mayoría de los problemas generados por las lesiones sólo con este método.

La ansiedad de la paciente o bien del médico tratante es una indicación que no se encuentra ampliamente validada. En nuestro estudio, en el único caso que se realizó por este motivo, la RM no tuvo mayor implicancia puesto que se evidenció hallazgos que si bien no eran sospechosos fueron susceptibles de generar más ansiedad. Sin embargo en los casos con resultados negativos es probable que este método de imagen sí contribuya a disminuir el nivel de preocupación.

Una indicación de gran utilidad, fuera de la esfera oncológica, fue la evaluación mamaria en pacientes con inyección libre de biopolímeros. En ellas no se evaluó cambio de conducta puesto que no existe consenso sobre su manejo. La acumulación de partículas de biopolímeros de muy alta densidad en mamografía y que en ecografía atenúan drásticamente el ultrasonido impiden una correcta visualización del parénquima mamario con estos métodos.

En dos de las pacientes en quienes no encontramos impregnaciones patológicas intraparenquimatosas, observamos que los biopolímeros se distribuyen en la periferia de las mamas, comprometiendo principalmente el celular subcutáneo, hecho que permitiría la detección de un eventual foco hipercaptante en el parénquima mamario.

En otras indicaciones como asimetría focal y la retracción cutánea sin otras alteraciones, la RM mamaria negativa permite un manejo expectante gracias a su alto VPN, al descartar un cáncer infiltrante o CDIS de alto grado asociado.

Cabe destacar que en nueve pacientes no fue posible realizar seguimiento, lo cual demuestra la necesidad de generar estudios prospectivos, sistematizados, que permitan, previo acuerdo del paciente, un expedito acceso a la información.

Por otra parte detectamos que en una proporción no despreciable de casos la resonancia 
está generando conductas clínicas sin evidencia científica, como el caso de manejo expectante de lesiones del tipo mucocele like o nódulos BI-RADS 4.

La constatación del porcentaje no menor de RM realizadas por indicaciones no habituales y la poca o nula evidencia científica existente para respaldar el cambio de conducta médica según los hallazgos del examen, nos deja como desafío la tarea de realizar estudios de costo-beneficio y seguimientos a largo plazo.

\section{Conclusión}

Evidenciamos que un porcentaje no despreciable (9.2\%) de RM mamarias realizadas en nuestro medio son por indicaciones distintas a las establecidas en consensos nacionales. En un $71.4 \%$ de los casos de este estudio, la RM mamaria determinó un cambio en la conducta médica, llevando en algunas ocasiones a efectuar manejo no avalado por la experiencia científica y que podría incluso ser riesgoso para la paciente. Estos resultados nos orientan a pensar que este examen podría ser útil en casos específicos y nos demuestran además la necesidad de estudios sistematizados, multicéntricos que lleven a reunir la evidencia suficiente para justificar o rechazar el uso de RM de mamas en indicaciones no habituales, distintas a las ya establecidas.

En casos excepcionales, en que parezca conveniente realizar este tipo de estudio, se recomienda generar discusiones y toma de decisiones por equipos multidisciplinarios.

\section{Bibliografía}

1. I. Doutriaux-Dumoulin, C.Digabel-Chabay, P. Meingan, S. Morand, C. Labbe-Develliers, M. Ricaud. Desorganisations architecturales / asymétries de densité: de la perception à la sémantique. Certitudes et incertitudes en senologie: de l'observation à l'action. SOFMIS 2005 3-4 juin 2005, Versailles.

2. Debra M. Ikeda. Magnetic Resonance Imaging of Breast Cancer and MRI- Guided Breast Biopsy. The requisites. Breast Imaging, 2004. 189-224.
3. Kriege M, Brekelmans CT, Boetes C, et al. Efficacy of $\mathrm{MRI}$ and mammography for breast-cancer screening in women with a familial or genetic predisposition. $\mathrm{N}$ Engl J Med 2004; 351: 427-37.

4. Robson, ME, Offit, K. Breast MRI for Women with Hereditary Cancer Risk. JAMA 2004; 292: 1368-1370.

5. Lee SG, Orel SG, Woo IJ, et al. MR imaging screening of the contralateral breast in patients with newly diagnosed breast cancer: preliminary results. Radiology 2003; 226: 773-778.

6. Morris EA, Liberman L, Ballon DJ, et al. MRI of occult breast carcinoma in a high-risk population. Am J Roentgenol AJR 2003; 181: 619-626.

7. Liberman L, Morris EA, Kim CM, et al. MR imaging findings in the contralateral breast of women with recently diagnosed breast cancer. Am J Roentgenol AJR 2003; 180: 333-341.

8. Bedrosian I, Schlencker J, Spitz FR, et al. Magnetic resonance imaging-guided biopsy of mammographically and clinically occult breast lesions. Ann Surg Oncol 2002; 9: 457-461.

9. Morris EA, Liberman L, Dershaw DD, et al. Preoperative MR imaging-guided needle localization of breast lesions. Am J Roentgenol AJR 2002; 178: 1211-1220.

10. Gilhuijs KG, Deurloo EE, Muller SH et al. Breast MR imaging in women at increased lifetime risk of breast cancer: clinical system for computerized assessment of breast lesions-initial results. Radiology 2002; 225: 907-916.

11. Brown J, Smith RC, Lee $\mathrm{CH}$. Incidental enhancing lesions found on MR imaging of the breast. Am J Roentgenol AJR 2001; 176: 1249-1254.

12. Orel SG, Schnall MD. MR imaging of the breast for the detection, diagnosis, and staging of breast cancer. Radiology 2001; 220: 13-30.

13. Fischer U, Kopka L, Grabbe E. Breast carcinoma: effect of preoperative contrast-enhanced MR imaging on the therapeutic approach. Radiology 1999; 213: 881-888.

14. Orel SG, Weinstein SP, Schnall MD, et al. Breast MR imaging in patients with axillary node metastases and unknown primary malignancy. Radiology 1999; 212: 543-549. 